\title{
DUST IN PLANETARY RING SYSTEMS
}

\author{
Mark R. Showalter \\ Center for Radar Astronomy \\ Stanford University \\ Stanford, CA 94305 \\ U.S.A.
}

\begin{abstract}
Each of the outer gas giants Jupiter, Satum, Uranus and Neptune is now known to be encircled by a system of rings. Some of these, such as the A, B, and C rings of Saturn and the nine narrow Uranian rings, are rather optically thick and are composed primarily of large bodies $(1 \mathrm{~cm}$ to $10 \mathrm{~m})$. However, every other system has been found to contain a large population of micron-sized dust. Such rings reveal the effects of a variety of physical processes that are also acting on interplanetary and interstellar grains. When such rings are examined as members of a general class, recurring patterns begin to emerge.
\end{abstract}

\section{Introduction}

Most of our knowledge about the diverse family of planetary rings comes from the recent reconnaissance of the outer planets by the Voyager spacecraft. This survey of the dusty rings will emphasize the current state of our knowledge about these rings' physical properties-what we know and how we know it. J. A. Burns presents in this volume a separate review of the dominant physical processes sculpting these rings.

For reasons that are not entirely clear, the known planetary rings show a nearly perfect anticorrelation between dust content and optical depth. This is undoubtedly related to the fact that dust tends to stick to larger particles, and so cannot survive for long in one of the denser rings. Hence, the study of dusty rings is synonymous with the study of the faintest planetary rings. Such rings are by their nature not always easy to detect, and so the data sets on some of these rings are quite limited in spite of the Voyager encounters. Indeed, some of the rings discussed below are only known because of a handful of Voyager observations, or in some cases just a single detection.

\section{Physical Properties of the Dusty Rings}

In this section I will present a very brief summary of the properties of the faint and dusty rings. Various general characteristics of these rings are also summarized in Table I.

A.C. Levasseur-Regourd and H. Hasegawa (eds.), Origin and Evolution of Interplanetary Dust, 349-356.

(C) 1991 Kluwer Academic Publishers, Printed in Japan. 


\subsection{JUPITER}

Jupiter's ring system was discovered in a single image from the Voyager 1 flyby in 1979, and subsequently imaged in greater detail by Voyager 2 . The most complete physical characterization of this ring system has been performed by Showalter et al. $(1985,1987)$. The ring appears to comprise three rather distinct components. The main ring is relatively thin and extends between orbital radii of 122,000 and $129,000 \mathrm{~km}$, where the outer boundary is far more abrupt than the inner. Showalter et al. (1987) find some evidence for three brighter features, of which two appear to be associated with the embedded satellites Adrastea and Metis. Photometry indicates that the dust particle size distribution is compatible with a power law. It appears that a population of larger bodies is also present, which are seen in backscatter and presumably act as source bodies for the dust (Burns et al., 1984).

Near the main ring's inner boundary arises the halo, a vertically extended cloud of material that seems to extend about halfway down to the top of Jupiter's atmosphere. This ring can be detected out to a full thickness of $\sim 30,000 \mathrm{~km}$. Its overall structure appears to be driven by resonant perturbations from Jupiter's inclined magnetic field (Burns et al., 1985). No evidence for macroscopic bodies can be found in this region.

Finally, Showalter et al. (1985) were the first to note the presence of an even fainter ring extending outward from the main ring in a single Voyager image. This, dubbed the "gossamer" ring, appears to have a peak near the location of synchronous orbit at $160,000 \mathrm{~km}$, which suggests a significant interaction with the local plasma. The ring does not have an abrupt outer boundary, but instead seems to fade out linearly, ending near the orbit of Thebe at $220,000 \mathrm{~km}$. The lack of images at multiple phase angles precludes any possible determination of the particle sizes.

\subsection{SATURN}

The innermost, or D ring of Saturn seems to occupy most of the region between the $\mathrm{C}$ ring and the planet's cloudtops. In the few Voyager images where it can be seen, this ring shows a remarkable amount of structure. Two narrow ringlets are visible, with a number of fainter belts of material interspersed. This data set has never received the closer scrutiny it deserves, and as yet much remains to be learned about this ring. However, the distinct brightening of the ring at the highest phase angles clearly indicates diffraction by micron-sized dust. Recently, Marley and Porco (1990) have proposed that some of the D ring's structure may be associated with resonances with Saturnian f-mode oscillations; this possibility clearly warrants closer examination.

Travelling outward, the next ring to show a preponderance of dust is a narrow ringlet near the middle of the Encke gap in the A ring. This ring reveals a great deal of longitudinal variability, and also some clumps and kinks reminiscent of the "braided" F ring (Smith et al., 1982). It is now known to share its orbit with the newly-discovered moon 1981S13 (Showalter, 1990). Once again, this ring has never received close scrutiny that it warrants, although an ample body of Voyager data exists. It is, however, clear that the ring brightens significantly at high phase angles, indicating a preponderance of dust.

The $\mathrm{F}$ ring itself was the first narrow and longitudinally variable ring observed (Smith $e t$ al., 1981, 1982). It was, at the time at least, the prototypical "shepherded" ring, since it appears to be confined by the two nearby satellites Pandora and Prometheus. Cuzzi and Burns (1988) find evidence for an additional belt of smaller moonlets in the vicinity, based on 
charged particle absorption signatures detected by Pioneer 11 . Photometry by Ockert et al. (1988) indicates that this ring has a narrow core of relatively high optical depth, along with a surrounding region composed primarily of dust. Furthermore, Burns et al. (1983) have found a very faint inward extension to this ring, which fills the region between the $F$ and $A$ rings.

Finally, Satum has two very faint outer rings, designated G and E. Interest in these two rings has been rekindled recently because of the potential hazard they may pose to the Cassini orbiter. Hence, the most complete analyses of these rings was performed by a variety of investigators specifically for the Cassini Project (Cuzzi et al., 1989). The isolated G ring was detected unambiguously in only two Voyager images, both at phase angles of $160^{\circ}$. It is roughly $7000 \mathrm{~km}$ wide, centered on a radius of $168,000 \mathrm{~km}$, and is not associated with any known moon. Recently, however, I have found faint traces of the ring in a number of images taken at a slightly lower phase angle. Strangely, the ring appears to be far narrower in these views, implying a core/halo configuration. The ring also shows significant longitudinal variability.

On the other hand, the E ring of Satum encompasses an area larger than that of all other planetary rings put together. It has a peak in density coincident with the orbit of the moon Enceladus, but encompasses the orbits of Mimas, Tethys and Dione as well. The ring is also quite thick, ranging from 10,000 to $50,000 \mathrm{~km}$ in vertical extent from its inner to its outer boundary. Showalter et al. (1991) combine a number of Earth- and Voyager-based observations to demonstrate that the ring contains a very narrow distribution of particle sizes, centered on roughly one micron. This narrow distribution precludes the possibility that the ring arises from collisional or disruptive processes, making it unlike any other known ring. Many investigators have proposed that the ring arises from "geysers" or "ice volcanos" directly from the surface of Enceladus.

\subsection{URANUS}

The nine "classical" Uranian rings have high optical depths and very little dust. One additional narrow ring discovered in the backscattered Voyager images (Smith et al., 1986), 1986U1R $(\lambda)$, has been found to be somewhat of an exception. In a single, very high phase Voyager image, this ring was found to be far brighter than any of its siblings, indicating that dust is a major constituent. Careful photometry by Ockert et al. (1987) has revealed this ring to have significant azimuthal variability.

The particular high-phase image noted above also reveals the presence of an extraordinary family of dust belts around and among the better known rings. Colwell and Esposito (1990) have developed models to describe how the neighboring rings can be both sources and sinks for this material.

Finally, one additional broad ring, designated $1986 \mathrm{U} 2 \mathrm{R}$, is visible in a single Voyager image at a $90^{\circ}$ phase angle. This ring is interior to all of the structure discussed above, with a peak at a radius of $38,000 \mathrm{~km}$ and a radial width of $\sim 5,000 \mathrm{~km}$. Unfortunately, very little can be determined about the particle properties of this ring from a single view, although a predominance of dust is strongly suspected.

\subsection{NEPTUNE}

The Voyager cameras revealed a number of new dusty rings in orbit about Neptune, in addition to helping settle the mysteries of the ring "arcs" originally detected from the ground. Smith $e t$ 
al. (1989) have designated four major ring components. Two relatively prominent narrow rings, $\mathrm{N} 53$ and $\mathrm{N} 63$, have radii of $62,900 \mathrm{~km}$ and $53,200 \mathrm{~km}$, respectively. Each of these lies roughly $1000 \mathrm{~km}$ beyond the orbit of a newly discovered satellite, and a dynamical association seems likely. It is the outer of these rings that harbors the three prominent arcs. In addition, a so-called "plateau" extends outward from N53 halfway to N63, with roughly constant opacity. Finally, N42 is a much fainter and broader ring at a radius of $41,900 \mathrm{~km}$, which does not have abrupt boundaries like the others. Very preliminary photometric modeling by Smith et al. indicates that all of these rings contain a mixture of dust and larger bodies representing comparable optical depths.

\section{Categorization of the Dusty Rings}

Table 1 provides a quick summary of the properties of all the rings discussed above. It becomes apparent that the rings show recurring patterns, that are likely to be an outward manifestation of common underlying processes. To pursue this further, I have identified four general sets of traits that seem to accommodate all of the rings described. Each is discussed briefly in turn below.

\subsection{CATEGORY 1: NARROW RINGS}

The category of narrow rings includes the Encke ringlet, $F$ ring and $G$ ring core at Saturn, the Uranian $\lambda$ ring and rings N53 and N63 of Neptune. Interestingly, all of these but N53 also show some evidence for longitudinal variability. The inverse observation, that all longitudinally variable rings are narrow, is not much of a surprise - a ring can only be variable if some force acts to counteract Kepler shear, and narrower rings have less shear to be counteracted. Nevertheless, this observation seems to suggest that such counteracting forces are quite prevalent, so that rings will generally clump if Kepler shear is sufficiently small.

It is likely not a coincidence that all of these rings except the $G$ ring have known moons nearby. The gravity of these moons probably provides the perturbations needed to confine clumps, although detailed mechanisms behind these effects are not always clear. These rings also tend to fall in the region around the planetary Roche limit, which is the place where mixtures of ring material and small moons are most common. The $G$ ring is particularly puzzling if its azimuthal variability is confirmed, since Van Allen (1983) has placed very firm limits on the mass of any moons in the vicinity.

Lastly, these particular rings all contain populations of larger bodies mixed in with the dust. It may be that dust production is merely a byproduct of the processes required to keep the larger bodies in these rings confined.

\subsection{CATEGORY 2: ONE-WAY EXTENSIONS}

This classification describes rings that extend in one preferred direction from a region of higher optical depth. It includes the Jovian halo, the inward extension to Saturn's F ring, several of the individual Uranian dust belts, and Neptune's "plateau" outside of N53. Most of these rings are believed to comprise dust evolving radially away from the denser source region in one preferred direction. However, it remains puzzling why some of these rings have an opposite boundary that is also relatively abrupt.

With the exception of Neptune's "plateau", all of these rings are composed of $100 \%$ dust. 
TABLE I. A general summary of the physical properties of dusty planetary rings.

\begin{tabular}{|c|c|c|c|c|}
\hline Optical & & Thickness & Width & Ring Properties* \\
\hline depth & dust & $(\mathrm{km})$ & $(\mathrm{km})$ & 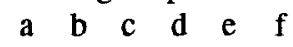 \\
\hline
\end{tabular}

\section{JUPITER}

Halo

Main Ring

"Gossamer"

$10^{-6}$
$3 \times 10^{-6}$
$10^{-7}$

100
$\sim 50$

$\begin{array}{cc}\sim 10,000 & 30,000 \\ \leq 300 & 7,000 \\ \leq 1,000 & 80,000\end{array}$

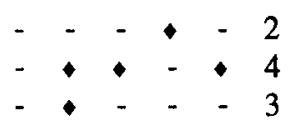

\section{SATURN}

D Ring

$<10^{-3} \quad 50-100$

$\leq 10$

Encke Ringlet

$0.1 \quad 100(?)$

$\leq 2$

$100-10,000$
20
3,000
$1-100$
7,000
$<1,000$
120,000

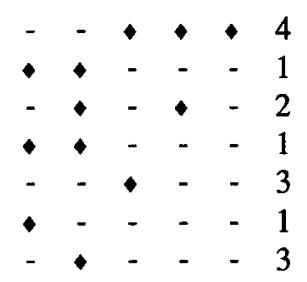

URANUS

1986U2R

Dust belts

1986U1R $(\lambda)$

$\begin{array}{cc}10^{-4}-10^{-3} & 100(?) \\ \leq 10^{-5} & 100(?) \\ 10^{-3} & >95\end{array}$

$\leq 100$

4,000

$\leq 100$

$\sim 10,000$

100

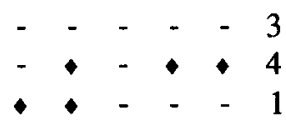

\section{NEPTUNE}

N42
N53
"Plateau"
N63

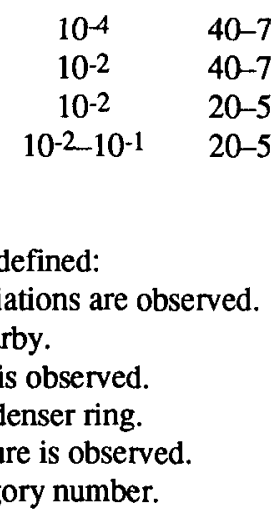

* Ring properties defined:
a: Azimuthal variations are observed.
b: Moons are nearby.
c: A denser core is observed.
d: Attached to a denser ring.
e: Internal structure is observed.
f: Assigned category number. 
This seems consistent with the general impression that nongravitational drag forces are behind the observed configuration. To be complete, I must also mention that two of the "classical" Uranian rings, $\delta$ and $\eta$, also show faint radial extensions. However, these are generally not composed of dust, and may have an entirely different explanation.

It is perhaps interesting to note that the extension's direction is nearly as often radially outward as inward, suggesting that a variety of drag forces are responsible for the evolution (see Burns et al., 1984). Also, one should recognize that the neighboring "source" region need only be denser in the relative sense-the Jovian halo is attached to a ring with $\tau \sim 10^{-6}$, whereas the Uranian dust belts are attached to rings with $\tau \geq 1$.

\subsection{CATEGORY 3: "FUZZY" RINGS}

This category includes the more diffuse rings, those lacking in any abrupt boundaries. The Jovian "gossamer" ring, the G and E rings of Satum, 1986U2R at Uranus and N42 at Neptune all seem to fit into this category. Such rings are often regarded as showing dust as it spreads away from a source region, and in that sense might be better called "two-way extensions" by analogy to Category 2. Like the rings in Category 2, these also tend to be composed entirely of dust (although N42 is an exception). However, the mechanisms behind this dispersal are not clear, and in fact the source regions are also unknown in several instances (e.g. 1986U1R, N42). Perhaps, very low optical depth belts of moonlets are hidden away in these regions.

It should be noted that no single drag force can explain these rings, in which material seems to evolve in both directions simultaneously. Mechanisms yielding a "random walk" may be required. Burns and Horanyi (1990) have come the furthest in explaining the peculiar radial structure of Saturn's E ring, by noting how solar perturbations can pump up the eccentricities of dust grains. In this case, then, the radial profile does not represent orbital evolution; it is merely the superposition of many grains on different eccentric orbits. However, it is unclear how relevant this particular mechanism is to the other rings in this category.

\subsection{CATEGORY 4: STRUCTURED RINGS}

Finally, I take note of the remaining dusty rings, which generally show more complex kinds of structure. Included are the main Jovian ring, along with Saturn's D ring and the family of Uranian dust belts. These rings all show structures at a wider variety of radial scales. They may be nothing more than hybrid versions of the previous categories, in which the dust distribution is built upon a more complex "skeleton" of source bodies and rings.

\subsection{OTHER RESULTS}

Surprisingly, the categorization just accomplished also reveals that some of the most obvious ring traits do not correlate with ring type. First, there is no obvious connection between category and ring optical depth. Although low optical depth may be required for the dust to survive, the actual value of $\tau$ does not seem to correlate with the underlying processes. Second, ring types do not correlate with planet. In fact, it is interesting to note that every planet has a ring from at least three of the four categories (despite the fact that Jupiter and Uranus only have three rings tabulated!). Finally, ring type does not correlate with particle composition (which tends to be silicates at Jupiter, water ice at Saturn, and carbonaceous material at Uranus and Neptune). 
The categorization I have outlined does have some predictive value, which can be tested as the rings described above are better understood. For example, the ratio of large bodies to dust in Neptune's N42 ring and plateau, based on the preliminary photometry of Smith et al. (1989), appears to be out of line with that expected for categories 2 and 3 . It will be interesting to perform a more complete analysis of the data and see if this discrepancy persists. Nevertheless, we have found that the outward similarities between the faint and dusty rings do seem to imply some common underlying processes.

\section{References}

Burns, J. A., J. N. Cuzzi, and M. R. Showalter (1983). Discovery of gossamer rings. Bull. Amer. Astron. Soc. 15, 1013-1014.

Burns, J. A., M. R. Showalter, and G. Morfill (1984). The ethereal rings of Jupiter and Saturn. In Planetary Rings (R. Greenberg and A. Brahic, Eds.), pp. 200-272. University of Arizona Press, Tucson.

Burns, J. A., L. E. Schaffer, R. J. Greenberg, and M. R. Showalter (1985). Lorentz resonances and the structure of the Jovian ring. Nature 316, 115-119.

Burns, J. A., and M. Horanyi (1990). Dynamics of the dust in Saturn's E ring. Bull. Amer. Astron. Soc. 22, 1042.

Colwell, J. E., and L. W. Esposito (1990). A numerical model of the Uranian dust rings. Icarus, in press.

Cuzzi, J. N., and J. A. Bums (1988). Charged particle depletion surrounding Satum's F ring: Evidence for a moonlet belt? Icarus 74, 284-324.

Cuzzi, J. N., J. F. Cooper, L. L. Hood, and M. R. Showalter (1989). Abundance and size distribution of ring material outside the main rings of Saturn. In Cassini Mission Proposal Package. JPL PD 699-11, 13, (5-1)-(5-F-7).

Marley, M. S., and C. C. Porco (1990). D ring features and f-mode oscillations of Saturn. Bull. Amer. Astron.Soc. 22, 1041.

Ockert, M. E., J. N. Cuzzi, C. C. Porco, and T. V. Johnson (1987). Uranian ring photometry: Results from Voyager 2. J. Geophys. Res. 92, 14969-14978.

Ockert, M. E., J. B. Pollack, and M. R. Showalter (1988). Voyager photometry of Satum's F ring II: the results. Bull Amer. Astron. Soc. $20,854$.

Showalter, M. R., J. A. Burns, J. N. Cuzzi, and J. B. Pollack (1985). The discovery of Jupiter's "gossamer" ring. Nature 316, 526-528.

Showalter, M. R., J. A. Bums, J. N. Cuzzi, and J. B. Pollack (1987). Jupiter's ring system: 
New results on structure and particle properties. Icarus $69,458-498$.

Showalter, M. R. (1990). Visual detection of 1981S13, the Encke gap moonlet. Bull. Amer. Astron. Soc. 22, 1040-1041.

Showalter, M. R., J. N. Cuzzi, and S. M. Larson (1991). Structure and particle properties of Saturn's E ring. Submitted.

Smith, B. A., et al. (1981). Encounter with Saturn: Voyager 1 imaging science results. Science 212, 163-191.

Smith, B. A., et al. (1982). A new look at the Saturn system: The Voyager 2 images. Science 215, 504-537.

Smith, B. A., et al. (1986). Voyager 2 in the Uranian system: Imaging science results. Science 233, 43-64.

Smith, B. A., et al. (1989). Voyager 2 at Neptune: Imaging science results. Science 246, $1422-1449$.

Van Allen, J. A. (1983). Absorption of energetic protons by Saturn's G ring. J. Geophys. Res. 88, 6911-6918. 